\title{
Patient Perspectives on Osseointegration: A National Survey of Veterans with Upper Limb
}

\section{Amputation}

\author{
Linda Resnik, $\mathrm{PhD}^{1,2^{*}}$, Heather Benz,PhD ${ }^{3}$ Matthew Borgia, $\mathrm{AM}^{1}$ Melissa A. Clark, $\mathrm{PhD}^{3,4}$
}

Running title: Patient Perspectives on Osseointegration

1Research Department,, Providence VA Medical Center, Providence, Rhode Island, United States of America

2Health Services, Policy and Practice, Brown University, Providence, Rhode Island, United States of America

3Center for Devices and Radiological Health, US Food \& Drug Administration, Silver Spring, Maryland, United States of America

4 Department of Quantitative Health Sciences, University of Massachusetts Medical school, Worcester Massachusetts, United States of America

*Corresponding Authorship:

E-mail: Linda.Resnik@,va.gov (LR)

\section{Funding Sources:}

U.S Army Medical Research Acquisition Activity W81XWH-16-2-0065

VA RR\&D A9264-S

\section{Acknowledgements}

This work was supported by the Office of the Assistant Secretary of Defense for Health Affairs, through the Orthotics and Prosthetics Outcomes Research Program Prosthetics Outcomes Research Award under Award No. W81XWH-16-0794 and through VA RR\&D A9265-S. Opinions, interpretations, conclusions and recommendations are those of the author and not necessarily endorsed by the Department of Veterans Affairs or the Department of Defense

\begin{tabular}{l} 
Abstract \\
\begin{tabular}{|l|} 
This article has been accepted for publication and undergone full peer review but has not \\
been through the copyediting, typesetting, pagination and proofreading process, which \\
may lead to differences between this version and the Version of Record. Please cite this \\
article as doi: 10.1111 pmrj.12147
\end{tabular} \\
\hline
\end{tabular}


Introduction: Osseointegrated (OI) prostheses have a unique benefit-risk profile among prosthetic alternatives and have been marketed in the U.S. under a Humanitarian Device Exemption since 2015. Information about upper limb prosthesis user perspectives on benefits and risks, prosthesis-user subpopulations for whom OI is most acceptable, and outcomes that matter most to patients could help inform clinical and regulatory decision-making. Recent $21^{\text {st }}$ Century Cures legislation expanded the role of patient experience data in the FDA decisionmaking process, recognizing that patient perspectives may be informative to regulators.

Objective: To better understand prosthesis user perspectives about the benefits and risks associated with upper limb OI prostheses.

Design: Patient perspective survey

Setting: Telephone administration

Participants: National sample of Veterans with upper limb loss.

Interventions: NA

Main Outcome Measures: Benefit-Risk survey developed for this study

Results: $28 \%$ of unilateral and $13 \%$ of bilateral amputees were willing to consider osseointegration surgery. Multivariate logistic regression models (OR; 95\%CI) showed that transhumeral amputation level (OR 1.40; 1.01-1.98) was associated with greater willingness to consider surgery, while older age (OR 0.17; 0.09-0.32) and higher VR-12 MCS (OR 0.53; 0.350.81) were associated with less willingness. Having a durable/reliable device, the ability to do more activities, and having a comfortable device were rated as very important or somewhat important by $98 \%$ or more for every risk condition

\section{Conclusions:}

Persons who were older, had transradial amputation (compared to transhumeral), and those who had better mental functioning were less willing to consider this surgery. Respondents who were willing to consider surgery indicated that the most important potential benefits were obtaining a durable/reliable device, the ability to do more activities, and having a comfortable device. Most were willing to accept one or more risks of surgery, with long term risks including chronic pain, loss of nerve function or device failure, considered the most unacceptable.

Keywords: Prostheses; Osseointegration; Upper limb amputation; Patient perspectives 


\section{Introduction}

Osseointegrated (OI) prostheses, which are attached to a residual limb by means of a fixture anchored in the bone, have a unique benefit-risk profile among prosthetic alternatives. They may improve user comfort, function, and sensation, but involve significant risks such as aseptic loosening (reported in $13-23 \%$ in upper extremity implants), periprosthetic fracture (reported in $0-18 \%$ of transfemoral implants, but $0 \%$ in upper extremity implants), intermedullary device breakage (reported in $27 \%$ of transradial implants) and infection (reported in 23-29\%) [1, 2]. They have been marketed in Europe since the 1990's, in Australia since 2011, and in the U.S. under a Humanitarian Device Exemption since 2015. While lower-limb OI surgery is available in the US through the Humanitarian Device Exemption, upper-limb OI surgery has not yet been FDA approved in the U.S.. However, clinical trials of upper-limb OI surgery are ongoing. OI has been a treatment option for individuals with lower and upper limb loss in Europe for over 20 years . [3]

Evidence has grown about the potential benefits and risks associated with OI prostheses [2], and the trade-offs between OI and other prostheses make the choice to pursue an OI prosthesis a shared decision between patients and healthcare professionals. Information about the diversity of upper limb prosthesis user perspectives on these distinctive benefits and risks, prosthesis-user subpopulations for whom OI is most acceptable, and the outcomes that matter most to patients could help inform clinical and regulatory decision-making.

Recent $21^{\text {st }}$ Century Cures legislation expanded the role of patient experience data in the FDA decision-making process, recognizing that the perspectives patients have about the impact of a condition and therapeutic options on their lives may be informative to regulators [4]. U.S. medical device approvals now include information about patient input considered during the 
approval process, and the impact of OI prostheses on quality of life is increasingly a part of the conversation about decision-making related to clinical use of these devices [5-7]. To better understand prosthesis user perspectives about the benefits and risks associated with upper limb OI prostheses, we developed a patient perspective survey and administered it to a national sample of Veterans with upper limb loss.

\section{Methods}

\section{Patient perspective survey development and pilot testing}

All aspects of this study were approved by appropriate institutional review boards and all participants gave informed consent. Development of the patient perspective survey involved three stages: development of an initial item set, prioritization of the survey content, and cognitive and pilot testing. Each stage is shown in Fig 1. Detailed description of survey development is provided in Appendix A. Briefly, in Stage A, semi-structured interviews were conducted with seven participants to understand the desired improvements in prosthetic technology that might motivate persons to incur risks to obtain new devices, and an initial item set was developed. As described in a previous manuscript [7], this stage resulted in lists of challenges and limitations related to function and quality of life faced by the respondents and desired improvements in prosthetic technology. Preliminary items addressing potential benefits and risks were then drafted by one of the authors (HB).

In Stage B, participants with upper limb difference prioritized the lists of benefits and risks for inclusion in the survey. This stage involved a point allocation exercise to prioritize the potential benefit-risk considerations, followed by a focus group, and a survey reported elsewhere [8]. During the focus group, participants discussed challenges they had experienced with amputation or limb difference, what they liked and disliked about the prostheses they had used, 
their experiences with pain, and what they would like to see in prostheses. Then they were asked to explain their responses to the prioritization exercise. Findings were used to refine items for inclusion in the benefit-risk survey. In Stage C, we tested potential participants' understanding of the item sets produced in Stage B and iteratively refined the items through cognitive testing and then pilot testing. Stage C resulted in the final version of the survey used in the national study. The final survey contained a yes/no screening question to ascertain participants' willingness to consider undergoing surgery to obtain a prosthesis that could restore sense of touch, provide more control over several types of prosthesis device movement, or eliminate the need for a prosthetic socket and harness, in addition to willingness to undergo specific risks associated with surgery. The benefit-risk items were only administered to those who answered yes to the screening question, indicating that they would consider undergoing surgery to obtain a particular benefit. The final version of the osseointegration survey section is shown in Appendix B. This manuscript reports on the data related to osseointegration. The results of benefit-risk questions about surgery to restore a sense of touch and provide more control over device movement will be reported elsewhere.

\section{Recruitment}

All Veterans with major upper limb amputation who received care in the VA between 2010-2016 (N=5639) were identified from VA Corporate Data Warehouse (CDW) sources. A total of 2288 persons were excluded (1479 deceased, and 601 missing valid addresses and/or phone numbers). Recruitment materials with opt out cards were sent to the remaining 3559 persons. Veterans who did not opt-out of participation $(\mathrm{N}=408)$ or inform us that they did not meet eligibility criteria $(\mathrm{N}=208)$ were contacted by telephone. Up to 10 phone call attempts were made. Veterans who were reached by phone $(\mathrm{N}=1893)$ were screened for eligibility. 


\section{Data Collection}

Eight hundred eight persons completed all or part of the larger national interview survey. This survey, described elsewhere [9], contained questions related to demographics, amputation level, laterality and etiology, prosthesis use, as well as standardized measures including the VR12 measure of health-related quality of life (HRQoL). The VR-12 is a Veteran version of the SF12 Health Survey that produces the Physical Component Summary (PCS) (Cronbach alpha in this sample $=0.86)$ and the Mental Component Summary (MCS) (Cronbach alpha in this sample $=0.88)$ scores. $[10,11]$

\section{Data Analyses}

We characterized demographics, amputation characteristics and prosthetic use for respondents. Descriptive analyses examined the proportion of respondents who indicated yes, no or not sure of their willingness to consider surgery for osseointegration by laterality of amputation (unilateral vs. bilateral), amputation level shoulder ( $\mathrm{SH}$ ), transhumeral (TH) and transradial (TR) gender, age group, category of HRQoL and etiology of amputation. We categorized HRQoL by separately grouping VR-12 PCS and MCS scores into three groups (low, medium, and high). The MCS and PCS have a population mean of 50 with a standard deviation (sd) of 10 (normative values). We considered those with scores more than $1 \mathrm{sd}$ below the mean on each scale to have low scores on that scale, and those within $1 \mathrm{sd}$ of the mean to have medium scores, and those with more than $1 \mathrm{sd}$ above the mean to have high scores.

We collapsed the categories of yes and not sure of willingness to consider surgery for osseointegration and used chi-square tests to examine bivariate relationships between key patient related variables (age category, unilateral/bilateral amputation, amputation level, gender, laterality of amputation, etiology of amputation) and willingness to consider surgery. To examine 
differences between sub-groups with three or more categories, we performed multiple comparison tests for variables using a Tukey-type method in SAS [12]. We then created a multivariate logistic regression model for willingness to consider surgery for osseointegration, including all variables that were significant in the bivariate analyses at $\mathrm{p}<0.10$.

For respondents who indicated a willingness (yes/unsure) to consider surgery, we examined ratings of importance of obtaining potential benefits (e.g. natural touch, better speed of movement control, ability to do more activities) given specific risks (e.g. overnight hospital stay, infection requiring antibiotics, infection requiring device removal). For respondents who indicated that they were willing to accept specific risks, we calculated the proportion who indicated that each of the specific benefits was important, somewhat important, or not important. We analyzed these data graphically to determine whether patterns of importance ratings varied by willingness to accept each type of risk.

We described the proportion of persons who were willing to accept each of the risks. We then compared ratings of benefit importance for each of the risks for prosthesis users and nonusers, and by amputation level graphically and using Fisher's exact tests.

\section{Results}

\section{Sampling frame and response rate-}

Eight hundred eight (83\%) of those screened to be eligible were successfully recruited into the study. The survey response rate and cooperation rate, calculated using the American Association for Public Opinion Research (AAPOR) methodology [13], was $47.7 \%$ and 63.3\%, respectively.

\section{<Insert Fig 2. Flow Diagram>}




\section{Demographic, amputation-related and prosthetic use characteristics}

The sample consisted of 776 unilateral amputees and 32 bilateral amputees (Table 2). Median age of the sample was 67.0 years (range 25 to 95); 764 (97.4\%) were male and 20 $(2.6 \%)$ were female. Table 1 shows characteristics of the sample. Seven hundred eighty-eight persons completed the entire interview, and 20 completed part but not the entire interview. Seventy-five percent of the sample identified as white, and $8.6 \%$ identified as Hispanic or Latino. Seventy percent of the sample were retired. Respondents had lost their limbs a median of 33.0 (range: 18.3 to 72.3 ) years prior to the interview. Amputation levels of respondents were $36.1 \%$ transradial (TR), 30.4\% transhumeral (TH), 16.2\% wrist joint (WD), 9.2\% shoulder disarticulation (SD), 5.2\% elbow disarticulation, and 3.0\% forequarter amputation (FQ). Accident was the most common cause of amputation (62.1\% unilateral, $62.5 \%$ bilateral), followed by "other" (54\% unilateral, and $71.9 \%$ bilateral), and combat injury (35.5\% unilateral, $28.1 \%$ bilateral). Burns were a prevalent cause of amputation for those with bilateral amputation (40.6\%). Most respondents were current prosthesis users (60\% unilateral, $84 \%$ bilateral respondents).

\section{<Insert Table 1. Demographics characteristics of unilateral and bilateral amputee respondents>}

\section{Willingness to Undergo Surgery}

Table 2 shows the proportion of respondents who indicated willingness to consider osseointegration surgery by sub-group characteristics. We found that $28.2 \%$ of unilateral and 
$12.9 \%$ of bilateral amputees were willing to consider osseointegration surgery, and $13.4 \%$ of unilateral and $12.9 \%$ of bilateral amputees were unsure.

\section{<Insert Table 2. Proportion of participants willing to consider osseointegration surgery >}

By amputation level, persons with TH amputation were the sub-group most likely to consider osseointegration surgery (35.0\% yes, $10.9 \%$ unsure). A smaller proportion of respondents who had lost their limbs due to combat injury, and a greater proportion of those who had lost their limbs due to accident indicated a willingness to undergo osseointegration surgery compared to persons with amputation from other etiologies.

Results of the bivariate analyses comparing participant characteristics associated with willingness to undergo surgery (yes or unsure vs. no) for each of the major benefits are shown in Table 3. Younger age, MCS category, and non-combat amputation were statistically associated with willingness to undergo osseointegration surgery. Post-hoc analyses revealed that there were statistically significant differences between the high and low and high and medium categories for MCS, with those with the highest category (best mental HrQoL) being less likely to be willing to consider surgery for osseointgration (results not shown). Post-hoc analyses by amputation level did not show any statistically significant differences by level (results not shown).

\section{<Insert Table 3 Bivariate comparisons of characteristics of participants by willingness to consider osseointegration>}

Multivariate logistic regression models that included all variables statistically significant at $\mathrm{p} \leq 0.10$ showed that age, amputation levels, and MCS were independently associated with willingness to consider surgery (Table 4). Specifically, the odds of being willing to consider 
surgery were 0.17 times lower for those 75 and older compared to those $18-45$ years. Relative to those with unilateral transradial level amputation, the odds of being willing to consider surgery were 1.40 higher for persons with unilateral transhumeral level amputation and 0.50 lower for those with bilateral amputation.

\section{<Insert Table 4. Multivariable logistic regressions for willingness to undergo surgery>}

Table 5 shows the proportion of respondents willing to accept each specific risk among those who were definitely or maybe willing to consider osseointegration surgery. Overall, long term risks, such as chronic pain, loss of some nerve function, or device failure requiring it to be removed, were unacceptable to the greatest proportion of respondents $(21.7 \%)$. Short-term restrictions on movement as well as pain and weakness for about a month were unacceptable to the smallest proportion (2.5\%). Forty-seven percent of respondents were willing and $40 \%$ were maybe willing to risk a serious infection that would require antibiotics.

\section{<Insert Table 5. Proportion of respondents willing to accept each surgical risk condition >}

Importance ratings of benefit factors were very similar across risk types. All potential benefits were considered very important or somewhat important for each risk condition by the majority of respondents. Having a durable/reliable device, the ability to do more activities, and having a comfortable device were rated as very important or somewhat important by $98 \%$ or more respondents for every risk condition. In contrast, naturalness of touch and water-resistance were the benefits rated not at all important by the greatest proportion of respondents $(7.3-8.1 \%$ and $4.2-5.5 \%$ respectively). As an example, Fig 3 shows the importance ratings of each potential 
benefit, given the risk of incurring an infection requiring IV antibiotics and hospitalization. Importance rating for all other risks are shown in Appendix C.

\section{$<$ Insert Fig 3 Ratings of importance of possible benefit for persons willing to risk infection requiring IV and Hospitalization>}

Comparison of importance ratings for each potential benefit-risk combination by amputation level showed differences at $\mathrm{p}<0.05$ for several benefits. We observed several statistically significant differences in importance rankings by amputation level. Although there were small differences in statistically significant findings, there was good consistency of findings across benefit-risk combinations by amputation level (Appendix C). As an example, for the risk of willingness to incur infection requiring IV antibiotics and an overnight hospital stay (Fig 4), there were differences in group rankings for the importance of more activities and durability/reliability of the device. The smallest proportion of persons with shoulder level amputation rated the ability to do more activities as very important. Although not statistically significantly different by sub-group in any risk condition, we also found that $100 \%$ of bilateral amputees rated water and dirt resistance and ability to lift more than $20 \mathrm{lbs}$. as very important in willingness to incur risk, and nearly $100 \%$ rated durability and reliability as very important. In contrast, the naturalness of touch was the benefit rated not at all important by the greatest proportion of respondents across all amputation levels in most every risk condition.

\footnotetext{
< Insert Fig 4. Sub-group comparisons of importance of factors for those who were willing to risk infection requiring antibiotics to obtain an osseointegrated prosthesis by amputation level>
} 
Comparisons of importance ratings for each potential benefit-risk combination by current prosthetic use did not show statistical differences except for the benefit of water/dirt resistance among those willing to consider long-term risks of pain or weakness during recovery (Appendix C). In these two comparisons, water/dirt resistance was more important to prosthetic users than to nonusers $(\mathrm{p}<0.05)$.

\section{DISCUSSION}

We conducted a first-of-its-kind national study that assessed upper limb amputee's willingness to consider osseointegration surgery. Our survey, limited to Veterans, also evaluated the importance of receiving specific benefits and examined whether these importance rankings varied by amputation level and for unilateral and bilateral amputees. Our survey demonstrates that amongst Veterans, there are a substantial proportion who would consider OI surgery should it be available. Our specific findings highlight those risks that are considered most and least acceptable, as well as the benefits most desired. This information is informative to researchers recruiting participants to OI trials and to clinicians who discuss the risks and benefits of OI with their patients. We found that $28 \%$ of unilateral and $13 \%$ of bilateral amputees were willing to consider osseointegration surgery, while a substantial proportion (approximately 13\%) were unsure. A greater proportion of respondents who were in older age categories, had bilateral amputation, and had better mental health functioning answered that they would not consider surgery. We found that persons with transhumeral amputation, a group with high abandonment rates [14], who may have difficulties with prosthesis socket fit, and thus are likely to receive the greatest benefit, were most likely to say they would consider this surgery (35\%). 
Our findings mirror those reported by Engdahl et al., who surveyed upper limb amputees about their interest in novel prosthetic interfaces but did not explicitly study osseointegration. They found that younger participants and those with unilateral amputation were more likely to be interested in the three invasive interfaces that they studied [15].

For those who indicated that they were or might be willing to consider osseointegration surgery, we examined whether ratings of benefit importance varied by specific risk conditions. The benefits that were rated least important across risks included natural touch, water/dirt resistant, and ability to lift more than $20 \mathrm{lbs}$. We found remarkable consistency in the ratings of benefits that were important across risks, with all potential benefits considered very important or somewhat important by most participants. This finding is not surprising because the list of potential benefits in our survey was identified through formative research with persons with amputation and other stakeholders.

Our study also quantified the acceptability of incurring a variety of post-surgical risks among those who indicated an openness to considering osseointegration surgery. All risks were acceptable or possibly acceptable to the majority of respondents who were willing or might be willing to consider osseointegration surgery. This demonstrates that persons who are open to considering surgery at all are willing to risk some adversity. Our results help sort these postsurgical risks by their perceived severity, with short term risks and 6 months of physical therapy the most acceptable and risk of bone breaking and long-term risks (including chronic pain, loss of some nerve function, or device failure requiring it to be removed) the most unacceptable. The risk of a serious infection that would require antibiotics was not a deterrent to most participants, with $87 \%$ of respondents willing or maybe willing to accept this risk. 
We found that ratings of benefit importance were consistent across all risk categories, but that persons with bilateral and shoulder level amputation, who are arguably more functionally impaired, weighed the importance of potential benefits directly related to function differently. Virtually all persons with bilateral amputation indicated that durability, water resistance, and lifting $>20$ lbs. were very important. In comparison, a greater proportion of persons with above and below elbow level amputation indicated that more activities and comfortable fit were very important.

We observed that persons categorized as having poor mental health were more likely to be willing to consider surgery for osseointegration. Further study is needed to disentangle the relationship between mental health and willingness or unwillingness to consider osseointegration surgery. We hypothesize that persons with poor prosthesis fit or who are uncomfortable about the appearance of their prosthesis may have poorer mental health and may be more open to considering interventions that can remediate their condition.

\section{Limitations:}

Our study had several limitations. First, it is possible that some participants did not fully understand what osseointegration surgery was. We did provide a brief explanation of osseointegration surgery, but we did not assess whether participants were familiar with this surgery or not, and we did not assess comprehension after the explanation was provided. Some may not have fully understood what the surgery entailed or appreciated how osseointegration would be used to attach the prosthesis. While osseointegration has been available in Europe for decades, most US amputees have not been exposed to this technology, and it is unlikely that they have ever met a person who had an osseointegrated limb.

Another limitation is that our sample included only Veterans who had received care at VA Medical Centers. These participants may not represent the larger population of Veterans or of US upper limb amputees more generally. However, we sampled $100 \%$ of Veterans who met our eligibility criteria and had a strong response rate, and thus our findings are likely 
generalizable to Veterans with upper limb amputation receiving healthcare at the VA. We believe that the findings are generalizable to both male and female Veterans. Although the Veteran sample is predominantly male, the response rate for females was high (62.8\% female, vs 47.3\% male). However, given the small number of females in the sample overall, there are limitations for generalizing from our findings of female Veterans to the civilian population..

Lastly, although we conducted statistical comparisons of importance for specific risk conditions, our analyses were limited due to small sample sizes for shoulder level and bilateral amputees, which may have resulted in insufficient power to detect small differences. Further research with larger samples and non-Veterans is needed to confirm or refute our findings.

\section{Conclusions}

We conducted a national survey of Veterans with major upper limb loss to assess their willingness to consider osseointegration and to understand their perspectives on osseointegration's potential benefits and risks. Twenty-eight percent of respondents were willing to consider osseointegration surgery. Persons who were older, had transradial amputation (compared to transhumeral), and those who had better mental functioning were less willing to consider this surgery. Respondents who were willing to consider surgery indicated that the most important potential benefits were having a durable/reliable device, the ability to do more activities, and having a comfortable device. Most were willing to accept one or more risks of surgery, with long term risks including chronic pain, loss of nerve function or device failure, considered the most unacceptable. 


\section{References}

1. Potter BK. From Bench to Bedside: A Perfect Fit? Osseointegration Can Improve Function for Patients with Amputations. Clin Orthop Relat Res. 2016;474(1):35-7.

2. Atallah R, Leijendekkers RA, Hoogeboom TJ, Frolke JP. Complications of boneanchored prostheses for individuals with an extremity amputation: A systematic review. PLoS One. 2018;13(8):e0201821.

3. Tillander J, Hagberg K, Hagberg L, Branemark R. Osseointegrated titanium implants for limb prostheses attachments: infectious complications. Clin Orthop Relat Res. 2010;468(10):2781-8.

4. Wilson H, Anatchkova M, Gelhorn H. A Perspective on the 21ST Century Cures Act: Patient-Focused Drug Development. 2017 [cited November]; 1-4]. Available from: https://www.evidera.com/wp-content/uploads/2017/11/04-A-Perspective-on-The-21st-CenturyCures-Act-Patient-Focused-Drug-Development_2017Nov-1.pdf.

5. Khemka A, Frossard L, Lord S, Bosley B, Muderis A, editors. Health-related quality of life of individuals with transfemoral amputation fitted with the Transcutaneous Bone Anchoring Prosthesis following the OGAAP. 6th International Conference Advances in Orthopaedic Osseointegration; 2015 March 26-27; Las Vegas, NV.

6. Hagberg K, Hansson E, Branemark R. Outcome of percutaneous osseointegrated prostheses for patients with unilateral transfemoral amputation at two-year follow-up. Arch Phys Med Rehabil. 2014;95(11):2120-7. Epub 2014/07/30.

7. Hansson E, Hagberg K, Cawson M, Brodtkorb TH. Patients with unilateral transfemoral amputation treated with a percutaneous osseointegrated prosthesis. The bone $\&$ joint journal. 2018;100-B(4):527-34.

8. Janssen EM, Benz HL, Tsai JH, Bridges JF. Identifying and prioritizing concerns associated with prosthetic devices for use in a benefit-risk assessment: a mixed-methods approach. Expert review of medical devices. 2018;15(5):385-98.

9. Resnik L, Ekerholm, S, Borgia, M, Clark, M. A National Study of Veterans with major upper limb amputation: Survey methods, participants, and summary findings. Under Review.

10. Ware J, Jr., Kosinski M, Keller SD. A 12-Item Short-Form Health Survey: construction of scales and preliminary tests of reliability and validity. Med Care. 1996;34(3):220-33.

11. Ware JE, Jr. SF-36 health survey update. Spine. 2000;25(24):3130-9. Epub 2000/12/22.

12. Elliot AC, Reisch JS. Implementing a Multiple Comparison Test for Proportions in a 2xc Crosstabulation in SAS ${ }^{\circledR}$. Center for Biostatistics and Clinical Science: UT Southwestern Medical Center, Dallas; Available from: http://www2.sas.com/proceedings/sugi31/204-31.pdf. 
13. Research. AAfPO. Response Rates - An Overview. 2018; Available from:

https://www.aapor.org/Education-Resources/For-Researchers/Poll-Survey-FAQ/Response-RatesAn-Overview.aspx.

14. Biddiss EA, Chau TT. Upper limb prosthesis use and abandonment: a survey of the last 25 years. Prosthet Orthot Int. 2007;31(3):236-57.

15. Engdahl SM, Chestek CA, Kelly B, Davis A, Gates DH. Factors associated with interest in novel interfaces for upper limb prosthesis control. PLoS One. 2017;12(8):e0182482. 


\begin{tabular}{|c|c|c|c|}
\hline & $\begin{array}{c}\text { Unilateral } \\
\text { Amputees } \\
\text { N=776 }\end{array}$ & $\begin{array}{c}\begin{array}{c}\text { Bilateral } \\
\text { Amputees }\end{array} \\
\mathbf{N}=\mathbf{3 2} \\
\end{array}$ & $\begin{array}{c}\text { All } \\
\mathrm{N}=808\end{array}$ \\
\hline & $\begin{array}{l}\text { Median } \\
\text { (range) }\end{array}$ & Median (range) & Median (range) \\
\hline Age (Years) & $\begin{array}{c}67.0(25.0, \\
93.0)\end{array}$ & $67.0(33.0,95.0)$ & $67.0(25.0,95.0)$ \\
\hline Missing (n) & $\mathrm{n}=24$ & $\mathrm{n}=0$ & $\mathrm{n}=24$ \\
\hline Years since initial amputation (either side) & $33.1(1.3,73.6)$ & $31.2(5.4,72.3)$ & $33.0(1.3,73.6)$ \\
\hline Years since amputation (second side) & NA & $29.8(5.4,72.3)$ & NA \\
\hline \multirow{2}{*}{ Missing (n) } & $\mathrm{n}=21$ & $\mathrm{n}=0$ & $\mathrm{n}=21$ \\
\hline & $\mathbf{N}(\%)$ & $\mathbf{N}(\%)$ & $\mathbf{N}(\%)$ \\
\hline \multicolumn{4}{|l|}{ Age category } \\
\hline 18,45 & $99(13.2)$ & $5(15.6)$ & $104(13.3)$ \\
\hline 45,65 & $207(27.5)$ & $9(28.1)$ & $216(27.6)$ \\
\hline 65,75 & $340(45.2)$ & $13(40.6)$ & $353(45.0)$ \\
\hline $75+$ & $106(14.1)$ & $5(15.6)$ & $111(14.2)$ \\
\hline Missing (n) & $\mathrm{n}=24$ & $\mathrm{n}=0$ & $\mathrm{n}=24$ \\
\hline \multicolumn{4}{|l|}{ Gender } \\
\hline Male & $755(97.3)$ & $32(100.0)$ & $787(97.4)$ \\
\hline Female & $21(2.7)$ & $0(0.0)$ & $21(2.6)$ \\
\hline Missing (n) & $\mathrm{n}=0$ & $\mathrm{n}=0$ & $\mathrm{n}=0$ \\
\hline \multicolumn{4}{|l|}{ Race } \\
\hline White & $583(77.5)$ & $22(68.8)$ & $605(74.9)$ \\
\hline Black & $86(11.4)$ & $3(9.4)$ & $89(11.0)$ \\
\hline Native American & $5(0.7)$ & $0(0.0)$ & $5(0.6)$ \\
\hline Other (including mixed race) & $30(4.0)$ & $4(12.5)$ & $34(4.2)$ \\
\hline Unknown & $48(6.4)$ & $3(9.4)$ & $75(9.3)$ \\
\hline Missing (n) & $\mathrm{n}=24$ & $\mathrm{n}=0$ & $\mathrm{n}=24$ \\
\hline \multicolumn{4}{|l|}{ Hispanic or Latino } \\
\hline Yes & $62(8.2)$ & $5(15.6)$ & $67(8.6)$ \\
\hline No & $678(90.2)$ & $26(81.3)$ & $704(89.8)$ \\
\hline Unknown & $12(1.6)$ & $1(3.1)$ & $13(1.7)$ \\
\hline Missing (n) & $\mathrm{n}=24$ & $\mathrm{n}=0$ & $\mathrm{n}=24$ \\
\hline \multicolumn{4}{|l|}{ Employment } \\
\hline Employed full-time & $73(9.7)$ & $1(3.1)$ & $74(9.4)$ \\
\hline Employed part-time & $31(4.1)$ & $13(40.6)$ & $31(4.0)$ \\
\hline Student & $20(2.7)$ & $0(0.0)$ & $20(2.6)$ \\
\hline
\end{tabular}




\begin{tabular}{|c|c|c|c|c|}
\hline Retired, but employed after amputation & $373(49.6)$ & \multicolumn{2}{|c|}{$13(40.6)$} & $386(49.2)$ \\
\hline Retired, but not employed after amputation & $152(20.2)$ & \multicolumn{2}{|c|}{$5(15.6)$} & $165(21.1)$ \\
\hline On medical leave & $9(1.2)$ & \multicolumn{2}{|c|}{$0(0.0)$} & $9(1.2)$ \\
\hline Other & $93(12.4)$ & \multicolumn{2}{|c|}{$0(0.0)$} & $98(12.5)$ \\
\hline Unknown & $1(0.1)$ & \multicolumn{2}{|c|}{$0(0.0)$} & $1(0.1)$ \\
\hline Missing (n) & $\mathrm{n}=24$ & \multicolumn{2}{|c|}{$\mathrm{n}=0$} & $\mathrm{n}=24$ \\
\hline \multicolumn{5}{|l|}{ Laterality of amputation } \\
\hline Unilateral Right & $370(47.7)$ & \multicolumn{2}{|c|}{$0(0.0)$} & $370(45.8)$ \\
\hline Unilateral left & $406(52.3)$ & \multicolumn{2}{|c|}{$0(0.0)$} & $406(50.3)$ \\
\hline Bilateral & $0(0.0)$ & \multicolumn{2}{|c|}{$32(100)}$. & $32(4.0)$ \\
\hline Missing (n) & $\mathrm{n}=0$ & \multicolumn{2}{|c|}{$\mathrm{n}=0$} & $\mathrm{n}=0$ \\
\hline \multicolumn{5}{|l|}{ Amputation Level } \\
\hline Forequarter & $23(3.0)$ & $1(3.1)$ & $0(0.0)$ & \\
\hline At the shoulder joint & $71(9.2)$ & $1(3.1)$ & $1(3.1)$ & \\
\hline Above the elbow & $236(30.4)$ & $5(15.6)$ & $4(12.5)$ & \\
\hline At the elbow & $40(5.2)$ & $\begin{array}{c}14 \\
(43.8) \\
\end{array}$ & $1(3.1)$ & \\
\hline Below the elbow & $280(36.1)$ & $\begin{array}{c}10 \\
(31.3) \\
\end{array}$ & $\begin{array}{c}20 \\
(62.5) \\
\end{array}$ & \\
\hline At the wrist joint & $126(16.2)$ & $0(0.0)$ & $6(18.8)$ & \\
\hline Through the hand & $0(0.0)$ & $1(3.1)$ & $0(0.0)$ & \\
\hline Missing $(\mathrm{n})$ & $\mathrm{n}=0$ & \multicolumn{2}{|c|}{$\mathrm{n}=0$} & \\
\hline \multicolumn{5}{|l|}{ Etiology of amputation } \\
\hline Combat injury & $275(35.5)$ & $9(28.1)$ & $9(28.1)$ & \\
\hline Accident & $481(62.1)$ & $\begin{array}{c}20 \\
(62.5) \\
\end{array}$ & $\begin{array}{c}20 \\
(62.5) \\
\end{array}$ & \\
\hline Burn & $81(10.5)$ & $\begin{array}{c}13 \\
(40.6)\end{array}$ & $\begin{array}{c}13 \\
(40.6) \\
\end{array}$ & \\
\hline Cancer & $30(3.9)$ & $0(0.0)$ & $0(0.0)$ & \\
\hline Diabetes & $11(1.4)$ & $0(0.0)$ & $1(3.1)$ & \\
\hline Infection & $86(11.1)$ & $9(28.1)$ & $8(25.0)$ & \\
\hline Other & $417(54.0)$ & $\begin{array}{c}21 \\
(65.6) \\
\end{array}$ & $\begin{array}{c}23 \\
(71.9)\end{array}$ & \\
\hline Missing (n) & $\mathrm{n}=0$ to 3 & $\mathrm{n}=0$ & $\mathrm{n}=0$ & \\
\hline
\end{tabular}

Table 1. Characteristics of unilateral and bilateral amputee respondents 


\begin{tabular}{|c|c|c|c|c|}
\hline & & \multicolumn{3}{|c|}{ Willing to consider osseointegration? } \\
\hline & $\mathbf{N}$ & Yes $(\mathrm{N}=215)$ & No $(N=461)$ & Not Sure $(N=104)$ \\
\hline \multicolumn{5}{|l|}{ Laterality } \\
\hline Unilateral & 776 & $211(28.2)$ & $438(58.5)$ & $100(13.4)$ \\
\hline Bilateral & 32 & $4(12.9)$ & $23(74.2)$ & $4(12.9)$ \\
\hline \multicolumn{5}{|l|}{ Amputation Level } \\
\hline Unilateral $\mathrm{SH}^{*}$ & 94 & $25(27.5)$ & $521(56.0)$ & $15(16.5)$ \\
\hline 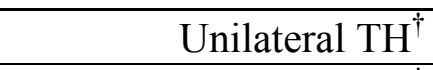 & 276 & $93(35.0)$ & $144(54.1)$ & $29(10.9)$ \\
\hline Unilateral $\mathrm{TR}^{\ddagger}$ & 406 & $93(23.7)$ & $243(62.0)$ & $56(14.3)$ \\
\hline Bilateral amputation & 32 & $4(12.9)$ & $23(74.2)$ & $4(12.9)$ \\
\hline \multicolumn{5}{|l|}{ Ever used prosthesis } \\
\hline Yes & 749 & $202(94.0)$ & $431(93.5)$ & $95(91.4)$ \\
\hline No & 52 & $12(5.6)$ & $29(6.3)$ & $9(8.7)$ \\
\hline Unknown & 2 & $1(0.5)$ & $1(0.2)$ & $0(0.0)$ \\
\hline \multicolumn{5}{|l|}{ Current prosthesis user } \\
\hline Yes & 490 & $120(59.4)$ & $290(67.8)$ & $63(66.3)$ \\
\hline No & 254 & $82(40.6)$ & $136(31.8)$ & $32(33.7)$ \\
\hline Unknown & 2 & $0(0.0)$ & $2(0.5)$ & $0(0.0)$ \\
\hline \multicolumn{5}{|l|}{ Ever used prosthesis } \\
\hline Yes & 749 & $202(94.4)$ & $431(93.7)$ & 95 (91.4) \\
\hline No & 52 & $12(5.6)$ & $29(6.3)$ & $9(8.7)$ \\
\hline \multicolumn{5}{|l|}{ Gender } \\
\hline Male & 764 & $208(96.7)$ & $450(97.6)$ & $102(98.1)$ \\
\hline Female & 20 & $7(35.0)$ & $11(55.0)$ & $2(10.0)$ \\
\hline \multicolumn{5}{|l|}{ Age } \\
\hline 18,45 & 104 & $41(39.4)$ & $45(43.3)$ & $18(17.3)$ \\
\hline 45,65 & 216 & $71(32.9)$ & $108(50.0)$ & $37(17.1)$ \\
\hline 65,75 & 353 & $87(24.8)$ & $220(62.7)$ & $44(12.5)$ \\
\hline $75+$ & 111 & $16(14.7)$ & $88(80.7)$ & $5(4.6)$ \\
\hline \multicolumn{5}{|l|}{$\mathbf{P C S}^{\S}$} \\
\hline Low & 234 & $68(29.4)$ & $131(56.7)$ & $32(13.9)$ \\
\hline Medium & 511 & $136(26.7)$ & $304(59.7)$ & $69(13.6)$ \\
\hline High & 13 & $3(23.1)$ & $10(76.9)$ & $0(0.0)$ \\
\hline \multicolumn{5}{|l|}{ MCS $^{\mathbb{R}}$} \\
\hline Low & 188 & $60(31.9)$ & $96(51.1)$ & $32(17.0)$ \\
\hline Medium & 344 & $100(29.3)$ & $196(57.5)$ & $45(13.2)$ \\
\hline High & 226 & $47(21.0)$ & $153(68.3)$ & $24(10.7)$ \\
\hline \multicolumn{5}{|l|}{ Etiology of amputation } \\
\hline \multicolumn{5}{|l|}{ Combat injury } \\
\hline 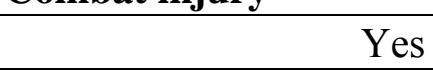 & 284 & $61(22.3)$ & $176(64.2)$ & $37(13.5)$ \\
\hline No & 523 & $154(30.4)$ & $285(56.3)$ & $67(13.2)$ \\
\hline Accident & & & & \\
\hline
\end{tabular}




\begin{tabular}{|lc|c|c|c|c|}
\hline & Yes & 501 & $137(28.1)$ & $280(57.5)$ & $70(14.4)$ \\
\hline No & 305 & $78(26.6)$ & $181(61.8)$ & $34(11.6)$ \\
\hline & & & & & \\
\hline Yes & 94 & $17(19.3)$ & $57(64.8)$ & $14(15.9)$ \\
\hline No & 711 & $198(28.6)$ & $404(58.4)$ & $90(13.0)$ \\
\hline & & & & & \\
\hline Yes & 30 & $8(28.6)$ & $18(64.3)$ & $2(7.1)$ \\
\hline No & 776 & $207(27.5)$ & $443(58.9)$ & $102(13.6)$ \\
\hline Infection & & & & & \\
\hline Yes & 12 & $6(50.0)$ & $5(41.7)$ & $1(8.3)$ \\
\hline No & 792 & $209(27.2)$ & $456(59.4)$ & $103(13.4)$ \\
\hline Other & & & & & \\
\hline Yes & 95 & $29(31.5)$ & $48(52.2)$ & $15(16.3)$ \\
\hline No & 709 & $186(27.0)$ & $413(60.0)$ & $89(12.9)$ \\
\hline
\end{tabular}

Table 2. Proportion of participants willing to consider osseointegration

$\mathrm{SH}=$ Shoulder disarticulation or forequarter

$\uparrow \mathrm{TH}=$ Transhumeral

$+\mathrm{TR}=$ Transradial

$\S$ PCS $=$ Physical Component Summary of the VR-12 Health Survey

IMCS=Mental Component Summary of the VR-12 Health Survey 


\begin{tabular}{|c|c|c|c|c|}
\hline & $\mathbf{N}$ & Yes /Maybe (N=319) & No $(N=461)$ & $\begin{array}{c}\text { Chisq/Fisher's } \\
\text { Exact p }\end{array}$ \\
\hline Amputation Level & & & & 0.062 \\
\hline Unilateral SH & 94 & $40(44.0)$ & $51(56.5)$ & \\
\hline Unilateral TH & 276 & $122(45.9)$ & $144(54.1)$ & \\
\hline Unilateral TR & 406 & $149(38.0)$ & $243(62.0)$ & \\
\hline Bilateral amputation & 32 & $8(25.8)$ & $23(74.2)$ & \\
\hline Laterality & & & & 0.081 \\
\hline Unilateral & 776 & $311(41.5)$ & $438(58.5)$ & \\
\hline Bilateral & 32 & $8(25.8)$ & $23(74.2)$ & \\
\hline Ever used prosthesis & & & & 0.943 \\
\hline Yes & 749 & $297(93.1)$ & $431(93.5)$ & \\
\hline No & 52 & $21(6.6)$ & $29(6.3)$ & \\
\hline Missing & 2 & $1(0.3)$ & $1(0.2)$ & \\
\hline Current prosthesis user & & & & 0.080 \\
\hline Yes & 490 & $183(61.6)$ & $200(67.8)$ & \\
\hline No & 254 & $114(36.4)$ & $136(31.8)$ & \\
\hline Missing & 2 & $0(0.0)$ & $2(0.5)$ & \\
\hline Gender & & & & 0.705 \\
\hline Male & 764 & $310(40.8)$ & $450(97.6)$ & \\
\hline Female & 20 & $9(45.0)$ & $11(55.0)$ & \\
\hline Age & & & & $<0.001$ \\
\hline 18,45 & 104 & $59(56.7)$ & $45(43.3)$ & \\
\hline 45,65 & 216 & $108(50.0)$ & $108(50.0)$ & \\
\hline 65,75 & 353 & $131(37.3)$ & $220(62.7)$ & \\
\hline $75+$ & 111 & $21(19.3)$ & $88(80.7)$ & \\
\hline PCS & & & & 0.311 \\
\hline Low & 234 & $100(43.3)$ & $131(56.7)$ & \\
\hline Medium & 511 & $205(40.3)$ & $304(59.7)$ & \\
\hline High & 13 & $3(23.1)$ & $10(76.9)$ & \\
\hline MCS & & & & 0.001 \\
\hline Low & 188 & $92(48.9)$ & $96(51.1)$ & \\
\hline Medium & 344 & $145(42.5)$ & $196(57.5)$ & \\
\hline High & 226 & $71(31.7)$ & $153(68.3)$ & \\
\hline Etiology of amputation & & & & \\
\hline Combat injury & & & & 0.032 \\
\hline Yes & 284 & $98(35.8)$ & $176(64.2)$ & \\
\hline No & 523 & $221(43.7)$ & $285(56.3)$ & \\
\hline Accident & & & & 0.239 \\
\hline Yes & 501 & $207(42.5)$ & $280(57.5)$ & \\
\hline No & 305 & $112(38.2)$ & $181(61.8)$ & \\
\hline Burn & & & & 0.251 \\
\hline
\end{tabular}




\begin{tabular}{|c|c|c|}
\hline & \multicolumn{2}{|c|}{ Osseointegration $(\mathrm{N}=753)$} \\
\hline & OR (95\% CI) & $\mathbf{p}$ \\
\hline \multicolumn{3}{|l|}{ Amputation Level } \\
\hline Unilateral SH & $1.19(0.73-1.95)$ & 0.488 \\
\hline Unilateral TH & $1.40(1.00-1.97)$ & 0.0 .050 \\
\hline Unilateral TR & (ref) & \\
\hline Bilateral amputation & $0.50(0.21-1.20)$ & 0.118 \\
\hline \multicolumn{3}{|l|}{ Age } \\
\hline 18,45 & (ref) & \\
\hline 45,65 & $0.57(0.34-0.96)$ & 0.033 \\
\hline 65,75 & $0.40(0.25-0.64)$ & $<0.001$ \\
\hline $75+$ & $0.17(0.09-0.32)$ & $<0.001$ \\
\hline \multicolumn{3}{|l|}{ MCS } \\
\hline Low & (ref) & \\
\hline Medium & $0.86(0.59-1.24)$ & 0.414 \\
\hline High & $0.53(0.35-0.81)$ & 0.003 \\
\hline \multicolumn{3}{|l|}{ Etiology of amputation } \\
\hline \multicolumn{3}{|l|}{ Combat injury } \\
\hline Yes & $0.68(0.44-1.07)$ & 0.093 \\
\hline No & (ref) & \\
\hline \multicolumn{3}{|l|}{ Accident } \\
\hline Yes & $1.01(0.67-1.54)$ & 0.952 \\
\hline \multirow{2}{*}{\multicolumn{3}{|c|}{ Infection }} \\
\hline & & \\
\hline Yes & $1.32(0.82-2.12)$ & 0.256 \\
\hline No & (ref) & \\
\hline
\end{tabular}

Table 4. Multivariable logistic regressions predicting willingness to consider osseointegration

$\mathrm{OR}=$ Odds Ratio

$\neq \mathrm{SH}=$ Shoulder disarticulation or forequarter

$\uparrow \mathrm{TH}=$ Transhumeral

$\$ \mathrm{TR}=$ Transradial

If MCS=Mental Component Summary of the VR-12 Health Survey 


\begin{tabular}{|c|c|c|c|}
\hline & \multicolumn{3}{|c|}{$\mathbf{N}=319$} \\
\hline & Yes & Maybe & No \\
\hline Risk condition & $\mathbf{N}(\%)$ & $\mathbf{N}(\%)$ & $\mathbf{N}(\%)$ \\
\hline Risk of infection that would require antibiotics & $149(47.2)$ & $126(39.9)$ & $41(13.0)$ \\
\hline $\begin{array}{l}\text { Risk of serious infection that would require } \\
\text { removing the device }\end{array}$ & $186(58.5)$ & $96(30.2)$ & $36(11.3)$ \\
\hline $\begin{array}{l}\text { Risk of bone breaking that would require surgery } \\
\text { to remove the device }\end{array}$ & $162(50.9)$ & $97(30.5)$ & $59(18.6)$ \\
\hline Long-term risks & $163(51.9)$ & $83(26.4)$ & $68(21.7)$ \\
\hline $\begin{array}{l}\text { Pain or weakness during the recovery from } \\
\text { surgery of about } 1 \text { month }\end{array}$ & $250(78.9)$ & $53(16.7)$ & $14(4.4)$ \\
\hline $\begin{array}{l}\text { Short-term restrictions on movement and } \\
\text { exercise for up to } 1 \text { month }\end{array}$ & $258(81.4)$ & $51(16.1)$ & $8(2.5)$ \\
\hline Up to 6 months of physical therapy & $261(82.3)$ & $47(14.8)$ & $9(2.8)$ \\
\hline
\end{tabular}

Table 5. Willingness to accept each surgical risk condition among those definitely or maybe willing to undergo osseointegration surgery 
Figure 2. Flow Diagram

Identified in VA Sample N=5639

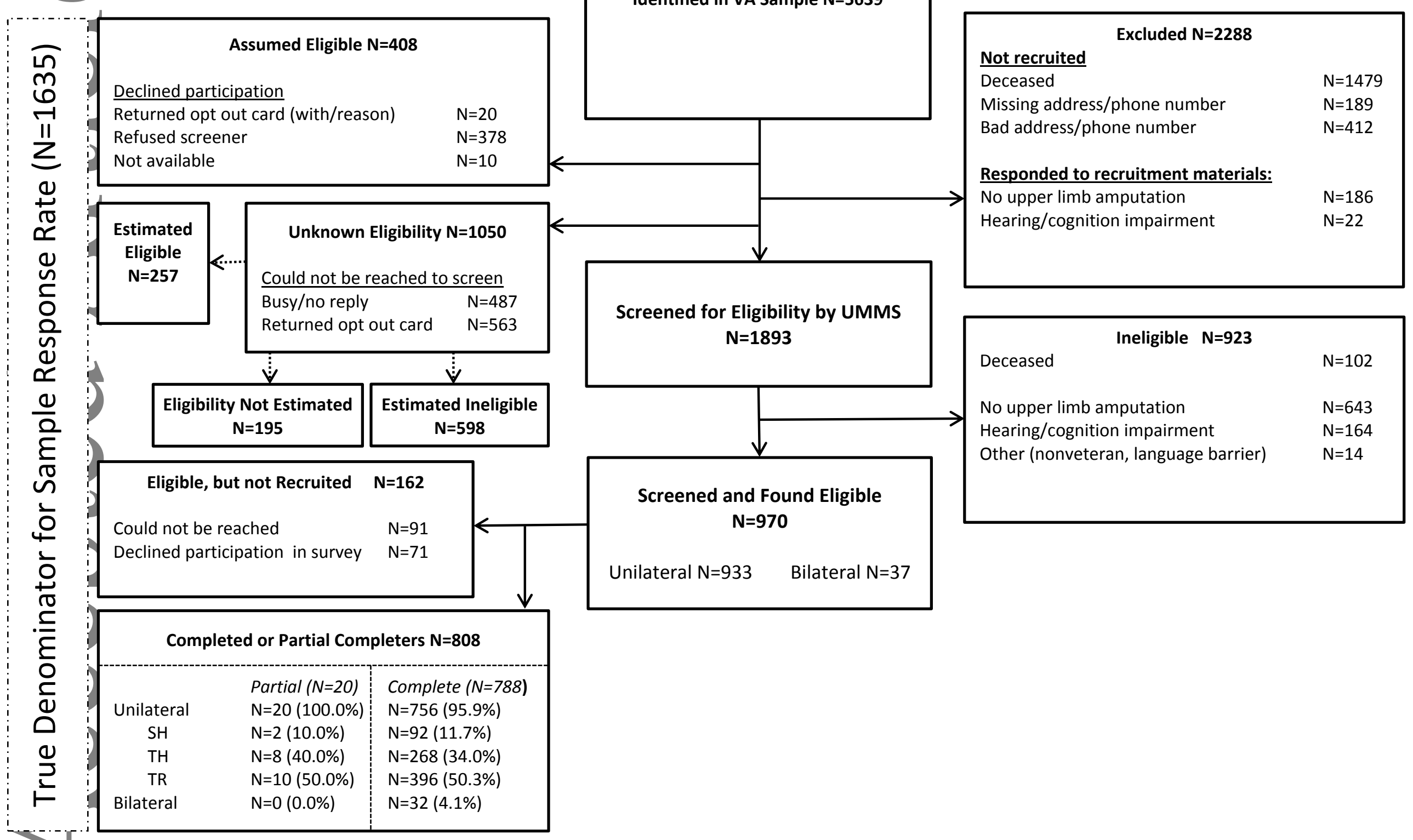




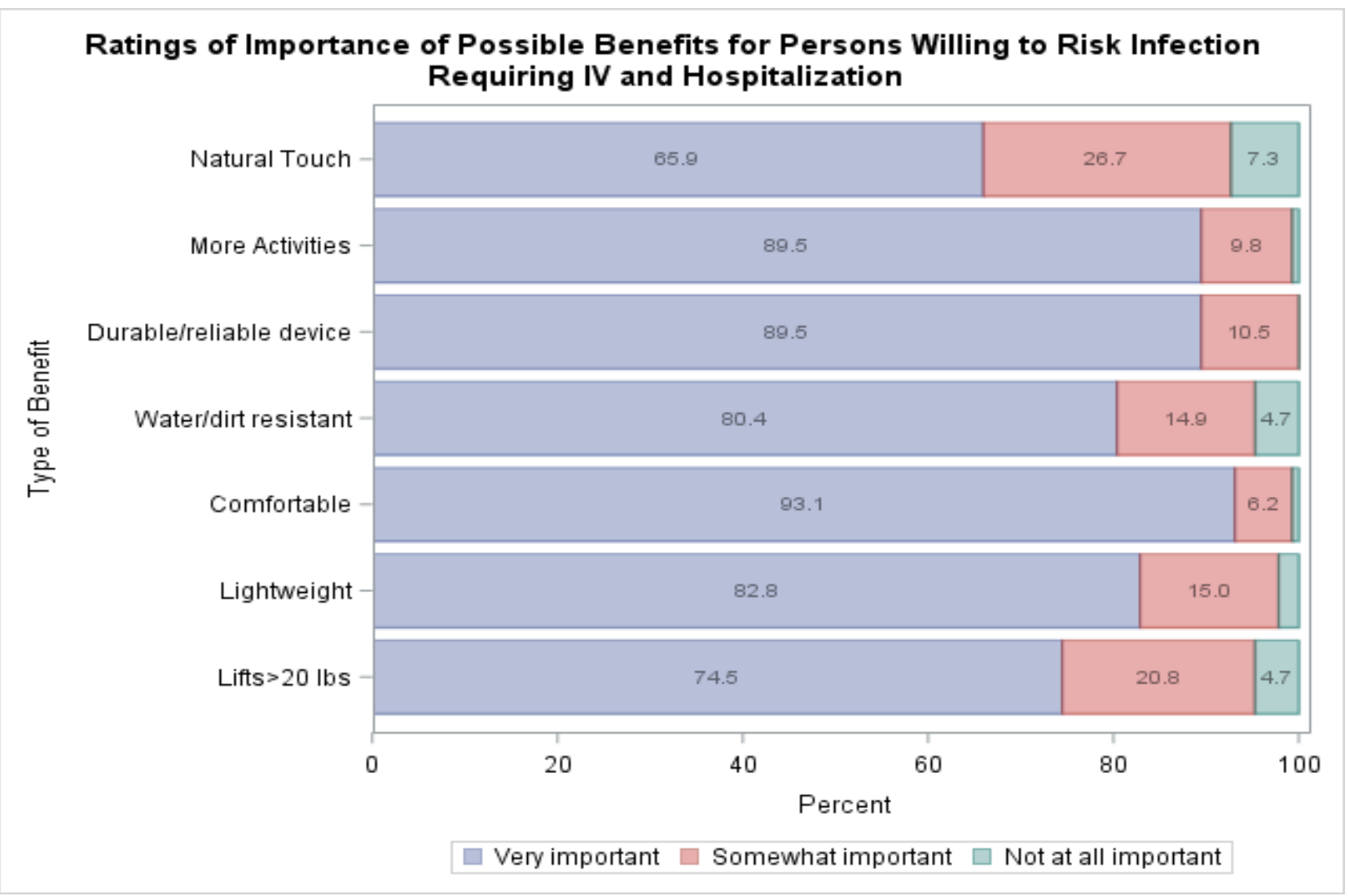

Figure 3. Benefit factor ratings for those who would consider Osseointegration surgery (yes/maybe): Example figure using those willing to risk infection requiring IV antibiotics and hospitalization) 
Willing to Risk: Infection Requiring IV and Hospitalization

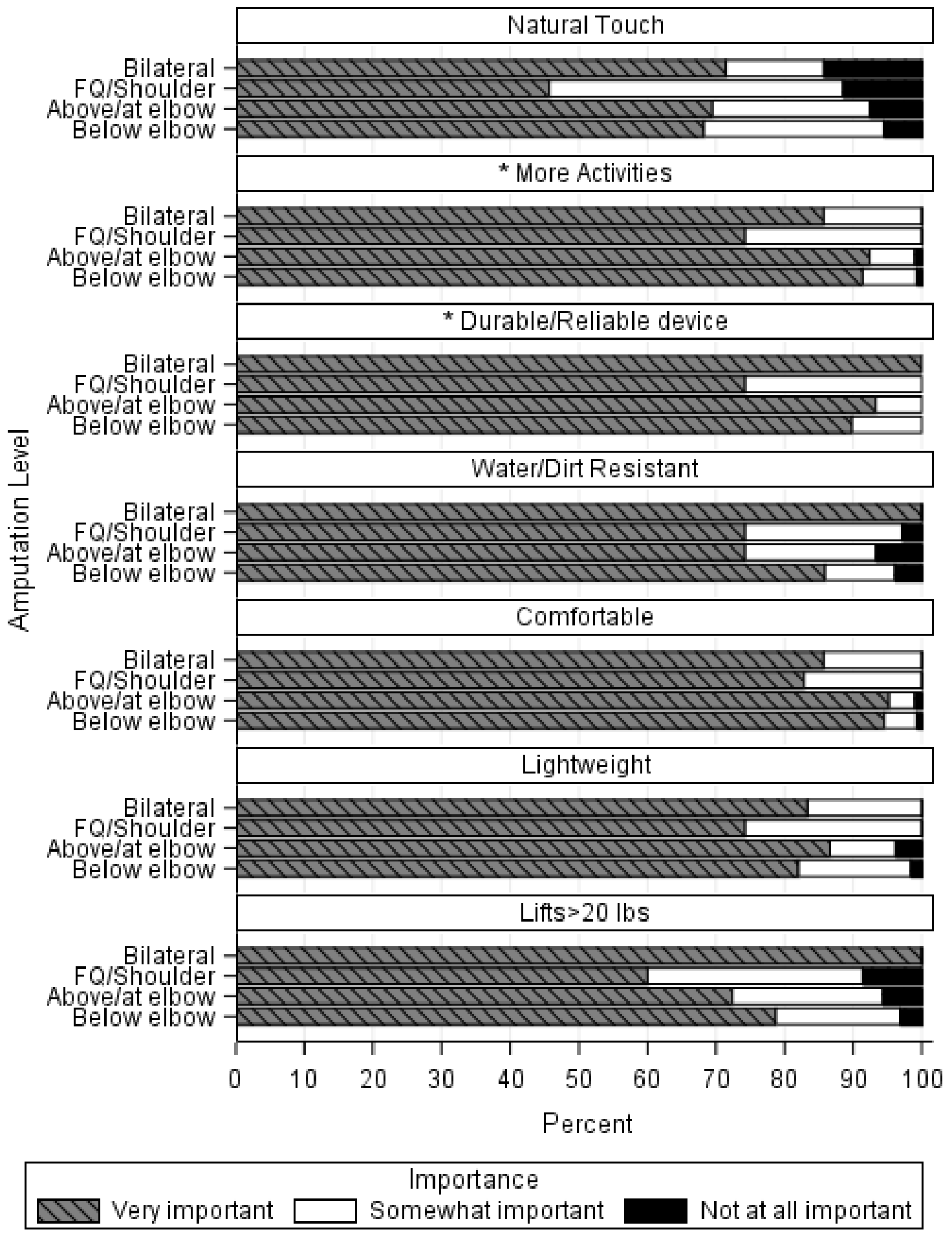

Fig 4. Sub-group comparisons of importance of factors for those who were willing to risks infection requiring antibiotics in order to obtain an osseointegrated prosthesis by amputation level

\footnotetext{
*significant at $\mathrm{p}<0.05$

$\mathrm{FQ}=$ Forequarter
} 